\title{
Derivation of Multiverse from Universal Wave Function Interpretation of String Theory
}

\author{
Dr. Zhi Gang Sha ${ }^{1}$ and Rulin Xiu ${ }^{2}$ \\ 1. Institute of Soul Healing and Enlightenment, 30 Wertheim Court, Unit 27D, Richmond Hill, Ontario L4B 1B9, Canada \\ 2. Hawaii Theoretical Physics Research Center, 16-266 E. Kipimana St, Keaau, HI 96749, USA
}

\begin{abstract}
The multiverse is a hypothesis created to solve certain problems in cosmology. Currently, this scheme is still largely ad hoc, rather than derived from fundamental laws and principles. Because of this, the predictive power of this theory is rather limited. Furthermore, there are concerns that this theory will make it impossible to calculate some measured quantities, such as the masses of quarks and the electron. In this paper, we will show that a new development in string theory, the universal wave function interpretation of string theory, provides a way to derive the mathematical expression of the multiverse. We will demonstrate that the Weyl invariance existing in string theory indicates that our observed universe is a projection from a hologram. We will present how the laws of physics can be derived from this fact. Furthermore, we suggest it may also provide a way to calculate the masses of fundamental particles such as quarks and the electron.
\end{abstract}

Key words: String theory, multiverse, grand unification theory, cosmology, holographic universe, universal wave function interpretation of string theory.

\section{Introduction}

The multiverse, also called meta-universe, parallel universes, and alternative universes, is a hypothetical set of possible universes, including the universe in which we live [1-4]. They determine the space, time, matter, energy, physical laws, and constants that describe them. The multiverse has been hypothesized in modern theoretical physics to solve many problems in cosmology, such as the cosmological constant problem. The solution to the cosmological constant problem would calculate and explain why the vacuum energy detected in our universe is much smaller than the normal theoretical calculation in quantum field theory.

Currently, the mathematical expression of the multiverse is usually obtained in an arbitrary way. The structure of the multiverse, the nature of each universe, and the relationships among the various constituent universes all depend on the specific multiverse

Corresponding author: Rulin Xiu, Ph.D., research field: theoretical physics. hypothesis considered. It is ad hoc, so to speak. This makes it impossible to experimentally test the validity of this theory $[5,6,7]$.

Another concern about the hypothesis of a multiverse is how a definite observation comes about and whether it is still possible to explain and calculate measured quantities such as the masses of quarks and the electron [8]. As Nobel laureate Steve Weinberg pointed out [8], if the multiverse existed, "the hope of finding a rational explanation for the precise values of quark masses and other constants of the standard model that we observe in our Big Bang is doomed, for their values would be an accident of the particular part of the multiverse in which we live".

In this paper, we explore how a new development in string theory, the UWFIST (universal wave function interpretation of string theory) can provide a solution to the above issues related to the multiverse hypothesis. We will demonstrate that within UWFIST, it is possible to derive the formula expressing the multiverses from fundamental principles. We will also show how the laws of physics, the equations of 
motion, and possibly the masses of fundamental particles can be derived in UWFIST.

String theory is a promising candidate for the grand unification theory [9-12]. How to understand and derive testable results from string theory has been a long debated subject. The currently accepted interpretation is that string theory is the study of the quantum dynamics of a string. The vibration of a string creates particles and interactions. When the scale of the string is set to the Planck Scale (approximately $1.6 \times 10^{-35}$ meters), string theory has the potential to predict the dimensions of space-time, the particle and interaction spectrum, to unify all the forces and fundamental particles, and more. However, with all of this great potential and promise, current string theory has not yet made many testable predictions.

In our previous work [11], by combining quantum physics and general relativity, we derive a space-time uncertainty relationship, which suggests that the wave function of the universe is created from a string like action. We call this the universal wave function interpretation of string theory. UWFIST indicates the existence of long-range quantum vibrations in our universe. These long-range vibrations could be the source of dark energy and the inflaton field. From the wave function of the universe, we can calculate or estimate the vacuum energy of our universe. In Ref. [11] we have demonstrated that using the age of our universe and fundamental constants such as the gravitational constant G, Planck constant $\hbar$, and speed of light, UWFIST may yield the estimate of vacuum energy consistent with the current experimental observation. We find that these large-scale quantum vibrations can cause and explain the observed large-scale anisotropies [12], anomalous alignments, and non-Gaussian distributions in the cosmic microwave background radiation as well as other background matter and radiation. It can also explain inflation and provide a -possible theoretical derivation for the inflaton and inflation scheme [13].
In this paper, we will briefly review the derivation of the UWFIST. We will show how to derive the mathematical expression of the multiverse and how to determine the observed physical laws, equations of motion, and potentially the mass and charge of fundamental particles from the UWFIST.

\section{Derivation of UWFIST and the Mathematical Expression of Multiverse}

In our previous work [11], we showed that when we took both the effects of quantum physics and gravity into consideration, we obtain an uncertainty relation between space measurement $\Delta \sigma$ and time measurement $\Delta \tau$ in the causal region:

$$
\Delta \sigma \Delta \tau \geq 1_{\mathrm{p}} \mathrm{t}_{\mathrm{p}}
$$

Here $l_{p}$ is the Planck length, $l_{p}=\left(\hbar G / c^{3}\right)^{1 / 2}=1.616 \times$ $10^{-35}$ meters, and $t_{p}$ is the Planck time, $t_{p}=\left(\hbar G / c^{5}\right)^{1 / 2}=$ $5.39 \times 10^{-44}$ seconds.

We suggest that this relation indicates that space and time are quantized and the non-commutation relation between space and time:

$$
[\sigma, \tau]=i 1_{\mathrm{p}} \mathrm{t}_{\mathrm{p}}
$$

Using the quantization procedure in quantum physics [14], we propose that the space time of uncertainty Eq. (1) and non-commutation Eq. (2) indicates a string action $\mathrm{A}_{\mathrm{s}}$ in the form:

$$
\mathrm{A}_{\mathrm{s}}=\int \mathrm{d} \tau \mathrm{d} \sigma / \mathrm{l}_{\mathrm{p}} \mathrm{t}_{\mathrm{p}}
$$

Action $\mathrm{A}_{\mathrm{s}}$ evolves the wave function from $\psi(0,0)$ at the space 0 and time 0 to $\psi(\mathrm{L}, \mathrm{T})$ at the space $\mathrm{L}$ and time $\mathrm{T}$ in the following way:

$$
\begin{aligned}
& \psi(\mathrm{L}, \mathrm{T})=\Sigma_{\text {sum over all possible paths }} \\
& \exp \left(\mathrm{i} \int_{0}^{\mathrm{T}} \mathrm{d} \tau \int_{0}^{\mathrm{L}} \mathrm{d} \sigma / \mathrm{l}_{\mathrm{p}} \mathrm{t}_{\mathrm{p}}\right) \psi(0,0)
\end{aligned}
$$

The Eq. (4) is the universal wave function interpretation of string theory.

UWFIST deviates from the usual interpretation of string theory in two ways. First, the world-sheet space $\sigma$ and time $\tau$ integration of the Eq. (3) is over the causal region i.e.

$$
A_{s}=\int_{0}{ }^{T} d \tau \int_{0}{ }^{L} d \sigma / l_{p}{ }^{2}
$$

Here $\mathrm{T}$ and $\mathrm{L}$ are the age and length of the causal horizon. They relate to the age and length of the 
horizon of our universe. In the normal interpretation of string theory, $\sigma$ is the string coordinate. $\mathrm{L}$ is taken to be the length of the string. It is set to be Planck length $1_{p}$. In the Eq. (4), $T$ and $L$ are variables. They change as universe evolves or as space and time pass by. The second difference between the usual string theory and UWFIST is that the universal wave function $\Psi(\mathrm{L}, \mathrm{T})$ is introduced. Here $\Psi(0,0)$ and $\Psi(\mathrm{L}$, $\mathrm{T})$ represent the universal wave function at the initial space and time and at the space $\mathrm{L}$ and time $\mathrm{T}$.

We propose that the universal wave function could be the wave function of our universe. If we extend the string action to the cases of superstring and heterotic string with background fields $[9,10]$, the universal wave function $\Psi$ can include all fundamental particles, all gauge interactions, gravity, and more. It also includes the large structures of our universe up to the scale of the horizon. In other words, UWFIST could be the wave function of our universe.

The wave function of our universe as derived in Eq. (4) shows the different possible universes. It is the mathematic expression of multiverse. UWFIST yields natural prediction, derivation, and mathematic formula of multiverse.

According to quantum physics, our observed universe is manifested by our measurement. Different measurements may yield different results. In the following, we will show that the Weyl invariance, generalized scale invariance may lead to the fixed universal constants and laws of physics, such as equations of motion and specific masses of fundamental particles. It can explain how the laws of physics and universal constants such as masses of quarks and the electron can come about in the world of the multiverse.

\section{The Universe Is a Projection from a Hologram}

In string theory, there are two sets of space-time: the two-dimensional world-sheet $(\sigma, \tau)$ and the observed space-time $\mathrm{X}^{\mu}(\sigma, \tau)$. The observed space-time $\mathrm{X}^{\mu}(\sigma, \tau)$ is a projection from the world sheet to possibly a higher dimensional space-time. In terms of $X^{\mu}(\sigma, \tau)$, the action $A_{s}$ in Eq. (3) becomes:

$$
\mathrm{A}_{\mathrm{s}}=\left(1 / \mathrm{l}_{\mathrm{p}} \mathrm{t}_{\mathrm{p}}\right) \int \mathrm{d} \tau \mathrm{d} \sigma \mathrm{g}^{1 / 2} \mathrm{~g}^{\mathrm{ab}} \partial \mathrm{aX} \mu \partial \mathrm{b} X^{\mu}
$$

Here $\mathrm{g}^{\alpha \beta}$ is the metric tensor on the world-sheet and $\mathrm{g}=-\operatorname{detg}^{\alpha \beta}$. As shown in string theory $[7,8]$, the general form of Eq. (6) in the presence of a massless background field is in the form:

$$
\begin{aligned}
\mathrm{A}_{\mathrm{s}}= & {\left[i \alpha \int _ { 0 } ^ { \mathrm { T } } \mathrm { d } \tau \int _ { 0 } ^ { \mathrm { L } } \mathrm { d } \sigma \mathrm { g } ^ { 1 / 2 } \left(\mathrm{g}^{\alpha \beta} \mathrm{G}^{\mu v} \partial_{\alpha} \mathrm{X}_{\mu} \partial_{\beta} \mathrm{X}_{v}\right.\right.} \\
& \left.\left.+\varepsilon^{\alpha \beta} \mathrm{B}^{\mu v} \partial_{\alpha} \mathrm{X}_{\mu} \partial_{\beta} \mathrm{X}_{v}+1 / 4 \alpha \Phi \mathrm{R}\right)\right]
\end{aligned}
$$

Here $\mathrm{G}^{\mu v}(\tau, \sigma), \mathrm{B}^{\mu v}(\tau, \sigma), \Phi(\tau, \sigma)$ are metric tensor, anti-symmetric tensor, and scalar background fields on the observed space. And R is the scalar curvature related to $\mathrm{g}^{\alpha \beta}$. The universal wave function in the presence of the background fields is:

$$
\begin{gathered}
\Psi\left(\mathrm{L}, \mathrm{T}, \mathrm{G}^{\mu v}(\mathrm{~L}, \mathrm{~T}), \mathrm{B}^{\mu v}(\mathrm{~L}, \mathrm{~T}), \Phi(\mathrm{L}, \mathrm{T})\right)= \\
\int \mathrm{DX}^{\mu} \mathrm{Dg}^{\mu v} \mathrm{DB}^{\mu v} \mathrm{D} \Phi \exp \left[\mathrm{i} \alpha \int_{0}^{\mathrm{T}} \mathrm{d} \tau \int_{0}^{\mathrm{L}} \mathrm{d} \sigma\right. \\
\left.\mathrm{g}^{1 / 2}\left(\mathrm{~g}^{\alpha \beta} \mathrm{G}^{\mu v} \partial_{\alpha} \mathrm{X}_{\mu} \partial_{\beta} \mathrm{X}_{v}+\varepsilon^{\alpha \beta} \mathrm{B}^{\mu v} \partial_{\alpha} X_{\mu} \partial_{\beta} X_{v}+1 / 4 \alpha \Phi \mathrm{R}\right)\right](8) \\
\text { We can extend } \Psi\left(\mathrm{X}^{\mu}(\mathrm{L}, \mathrm{T}), \mathrm{G}^{\mu v}(\mathrm{~L}, \mathrm{~T}), \mathrm{B}^{\mu v}(\mathrm{~L}, \mathrm{~T}),\right.
\end{gathered}
$$
$\Phi(\mathrm{L}, \mathrm{T}))$ to include other massive vibrations. We can also extend the above string action to the cases of superstring and heterotic string to include all fundamental particles; all gauge interactions, gravity, and more. This universal wave function tells us the probability for our universe to be at space-time $(\mathrm{L}, \mathrm{T})$ and background fields $\mathrm{G}^{\mu v}(\mathrm{~L}, \mathrm{~T}), \mathrm{B}^{\mu v}(\mathrm{~L}, \mathrm{~T}), \Phi(\mathrm{L}, \mathrm{T})$. We can do a Fourier transformation to transform the wave function $\Psi(\mathrm{L}, \mathrm{T}), \mathrm{G}^{\mu v}\left(\mathrm{~L}, \mathrm{~T}, \mathrm{~B}^{\mu v}(\mathrm{~L}, \mathrm{~T}), \Phi(\mathrm{L}, \mathrm{T})\right)$ into the vibrational space expressed by the various vibrations $\left(\omega^{\mu}, \kappa^{\mu \nu}, \ldots\right)$. The wave function $\Psi\left(\omega^{\mu}(L, T)\right.$, $\left.\kappa^{\mu v}(L, T), \ldots\right)$ expresses what kind of vibrations exist in our universe and how much they exist at proper time $\mathrm{T}$ and horizon length $\mathrm{L}$.

The proper space $\sigma$ and time $\tau$ form the world-sheet. The world-sheet has the Weyl symmetry, the local rescaling invariance of the world-sheet metric. The Eq. (7) is invariant under the local rescaling of the world-sheet metric:

$$
\begin{aligned}
& \mathrm{g}^{, \alpha \beta}(\tau, \sigma)=\exp (2 \omega(\tau, \sigma)) \mathrm{g}^{\alpha \beta}(\tau, \sigma), \\
& \mathrm{X}^{, \mu}(\tau, \sigma)=\mathrm{X} \mu(\tau, \sigma), \\
& \mathrm{G}^{, \mu v}(\tau, \sigma)=\mathrm{G}^{\mu v}(\tau, \sigma),
\end{aligned}
$$




$$
\begin{aligned}
& \mathrm{B}^{\mu \nu}(\tau, \sigma)=\mathrm{B}^{\mu v}(\tau, \sigma), \\
& \Phi^{\prime}(\tau, \sigma)=\Phi(\tau, \sigma),
\end{aligned}
$$

for an arbitrary $\omega(\tau, \sigma)$.

The Weyl invariance of the world-sheet indicates that if we expand, contract, or rotate the world-sheet, the world-sheet will look the same. In other words, the world-sheet is a hologram. Our observed space-time $\mathrm{X}^{\mu}(\tau, \sigma)$, particles, gauge and gravity interactions are the projection from this hologram. In other words, the observed universe is a projection from the world-sheet hologram.

\section{Derivation of the Laws of Physics and Equations of Motion}

The fact that our observed space-time is a projection from a hologram has great consequences. One of the results is the classical equation of motion regarding the background field $\mathrm{G}^{\mu \nu}, \mathrm{B}^{\mu \nu}, \Phi$, and more expanded as the functions of $X^{\mu}[1,2]$. The detailed derivation is presented in Refs. $[1,2]$. We will just summarize this derivation. In a nutshell, it is obtained by calculating the higher order corrections to the Eq. (7). The Weyl invariance is automatically preserved at the first order. However, the higher order corrections threaten to violate it. For example, at the second order, the following corrections occur:

$$
\begin{aligned}
& \beta_{\mu \nu}^{\mathrm{G}}=\alpha \mathrm{R}_{\mu \nu}+ 2 \alpha \nabla_{\mu} \nabla_{v} \Phi+\alpha / 4 \mathrm{H}_{\mu \lambda \omega} \mathrm{H}_{\nu}^{\lambda \omega}+\mathrm{O}\left(\alpha^{2}\right) \\
& \beta_{\mu \nu}^{\mathrm{B}}=--\alpha / 4 \nabla^{\omega} \mathrm{H}_{\mu \lambda \omega}+\alpha \nabla^{\omega} \Phi \mathrm{H}_{\omega \mu \nu}+\mathrm{O}\left(\alpha^{2}\right) \\
& \beta^{\Phi}=(\mathrm{D}-26) / 4-\alpha / 2 \nabla^{2} \Phi+\alpha \nabla^{\omega} \Phi \nabla_{\omega} \Phi- \\
& \alpha / 24 \mathrm{H}_{\mu \nu \lambda} \mathrm{H}^{\mu \nu \lambda}+\mathrm{O}\left(\alpha^{2}\right)
\end{aligned}
$$

The preservation of Weyl invariance at the higher orders requires that:

$$
\beta_{\mu \nu}^{\mathrm{G}}=\beta_{\mu \nu}^{\mathrm{B}}=\beta^{\Phi}=0 .
$$

Notice that $\beta_{\mu \nu}^{\mathrm{G}}=0$ leads to the generalized Einstein's equation with the source terms from the anti-symmetric tensor and dilaton. The equation $\beta^{\mathrm{B}}{ }_{\mu \nu}=$ 0 is the antisymmetric generalization of Maxwell equation. We can see that by requiring Weyl invariance, we are able to derive the equation of motion for the background fields $\mathrm{G}^{\mu \nu}, \mathrm{B}^{\mu \nu}$, and $\Phi$. In other words, the classical equations of motion including Einstein's general relativity, electromagnetism, and other gauge interactions, fundamental particles, and other background fields and matter come from the fact that our universe is a projection from a hologram. This can explain how fundamental laws such as Einstein's general relativity, electromagnetism, and other gauge interaction, fundamental particles, and other background fields and matter come about in the multiverse.

\section{Derivation of the Masses of Fundamental Particles from UWFIST}

Particle physics studies the fundamental particles that make up matter in the physical realm. Electrons, muons, quarks, and photons are some of these elementary particles. One of the fascinating phenomena discovered in particle physics is that particles also have "generations". Physicists have discovered three generations of elementary particles so far. The different generations have similar sets of elementary particles, except that their masses are different.

The Higgs boson is the current scheme to give mass to elementary particles in the standard model. We suggest that by requiring the Weyl invariance at higher order, we may obtain further restrictions or equations to gravity, electromagnetism, and other gauge interaction, fundamental particles, and other background fields and forms of matter. This may lead to the determination of the mass of some particles. The masses are the possible solutions to these equations. This may provide a way for us to derive the masses of fundamental particles, and may possibly explain the existence of generations of elementary particles.

\section{Conclusion}

In this paper, we show that the multiverse is a natural result of UWFIST. UWFIST not only supports the multiverse hypothesis, it also provides the scheme to mathematically derive the expression of the multiverse from fundamental principles by providing 
a way to calculate the wave function of our universe. The wave function of our universe exposes mathematically countless possible universes existing simultaneously. Our universe actually contains many possibilities. It exists simultaneously in all these possibilities. In this sense, we exist in multiple universes.

In the myriad of the multiverse, our world changes constantly and quickly depending on our observations and measurements. However, certain things do not change due to the fact that our universe is a projection from a hologram. The unchanging quantities make up the laws and specific matter we observe. The existence of classical equations as well as definite masses and possibly other properties are due to the fact that our universe is a projection from a hologram. It has already been shown in string theory that Weyl invariance in string theory leads to the observed laws of physics and the equations of motion. We suggest that it may also determine the specific masses of the observed fundamental particles.

We suggest one should calculate higher order corrections of Weyl invariance to determine the masses of the observed fundamental particles. We will present our work on this in the future.

\section{References}

[1] Kragh, H. 2009. "Contemporary History of Cosmology and the Controversy over the Multiverse." Annals of Science 66 (4): 529-51.

[2] Steinhardt, Paul. 2014. "Big Bang Blunder Bursts the Multiverse Bubble." Nature 510: 9.

[3] Max Tegmark, and Alexander Vilenkin. 2011. "The Case for Parallel Universes.” Retrieved 12 October 2013.
[4] Feeney, Stephen M., et al. 2011. "First Observational Tests of Eternal Inflation: Analysis Methods and WMAP 7-Year Results.” Physical Review D 84 (4): 43507.

[5] Ellis, George, and Silk, Joe. 2014. "Scientific Method: Defend the Integrity of Physics." Nature 516: 321-3

[6] Ellis George, F. R. 2011. "Does the Multiverse Really Exist?" Scientific American. Nature Publishing Group. 305 (2): 38-43. ISSN 0036-8733.

[7] Baggott, Jim. 2013. Farewell to Reality: How Modern Physics Has Betrayed the Search for Scientific Truth. Pegasus. ISBN 978-1-60598-472-8.

[8] Weinberg, Steven. 2007. Physics: What We Do and Do Not Know. The New York Review of Books.

[9] Michael Green, John H. Schwarz, and Edward Witten. 1987. Superstring Theory. Cambridge University Press. Vol. 1: Introduction. ISBN 0-521-35752-7. Vol. 2: Loop Amplitudes, Anomalies and Phenomenology. ISBN 0-521-35753-5.

[10] Polchinski, Joseph. 1998. String Theory. Cambridge University Press. Vol. 1: An Introduction to the Bosonic String. ISBN 0-521-63303-6. Vol. 2: Superstring Theory and Beyond. ISBN 0-521-63304-4.

[11] Sha, Z. G., and Xiu, R. L. 2017. "Dark Energy and Estimate of Cosmological Constant from String Theory." Journal of Astrophysics \& Aerospace Technology accepted on Mar 27, 2017

[12] Sha, Z. G., and Xiu, R. L. 2017. "Derivation of Large-Scale Anisotropy and Anomalous Alignment from Universal Wave Function Interpretation of String Theory." Journal of Astrophysics \& Aerospace Technology on May 06,2017

[13] Sha, Z. G., and Xiu, R. L. 2017. "Inflation Scheme Derived from Universal Wave Function Interpretation of String Theory." Journal of Astrophysics \& Aerospace Technology accepted on June 27, 2017

[14] Feynman, R. P., and Hibbs, A. 1965. Quantum Mechanics and Path Integrals. McGraw Hill. ISBN 0-07-020650-3. 